International Journal of Aviation Science and Technology

\title{
Monitoring System of Vibroacoustic Parameters of a Working Zone
}

\author{
Stakhova Anzhelika ${ }^{1 *}$, \\ ${ }^{1}$ National Aviation University, Kyiv/Ukraine \\ sap@nau.edu.ua - (D) 0000-0001-5171-6330
}

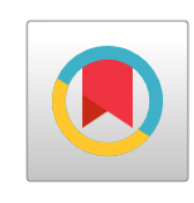

\begin{abstract}
This article discusses the safety problems of the use of aviation technology associated with the influence of operational vibration of aircraft. The topical issue of timely detection and prevention of a dangerous state of critical machines and mechanisms is analyzed. Modern means of measuring vibration parameters, principles of measurement, as well as characteristics of the sensitive element of the measuring transducer, are considered. The block diagram and operation algorithm of the proposed system for monitoring vibroacoustic parameters, which is built on the basis of a piezoelectric transducer, is presented. This system can measure the parameters of noise and vibration and analyze the measured data, signal about exceeding the permissible ranges for human work, display the measured data. The advantage of the proposed system is the connection of the measuring channels with the mainboard using the Bluetooth module, which allows the sensors to measure noise and vibration to be placed in any part of the working area.
\end{abstract}

\section{Keywords \\ Vibration \\ Noise \\ Measurement \\ System \\ Vibration sensors}

Time Scale of Article

Received 6 August 2021

Revised until 28 October 2021

Accepted 2 November 2021

Online date 24 December 2021

\section{Introduction}

At present, the state of flight safety remains at a rather low level, since every year there are aviation accidents with the loss of equipment and human lives. Flight safety is largely determined by the reliability of all units (Tomaszek H et al., 2010). Modern aircraft have a large number of units, devices, systems, instruments, with the help of which automatic and automated control of steering surfaces, landing gear, power plant, communication and navigation facilities, radar, life support systems for the crew and passengers, etc. is carried out (Advanced Avionics Handbook). The complexity and importance of the tasks solved with the help of the aircraft include the automation of onboard systems with a high degree of survivability, safety, reliability, and efficiency of operation. Along with the quantitative growth and expansion of application opportunities, aviation science and industry are faced with the task of significantly improving the technical and economic characteristics of aircraft.

Improvement of control systems of modern aircraft with various automatic devices posed difficult tasks for designers to ensure flight safety in the event of a failure of these devices. The aircraft control system must provide control in all flight modes with the required accuracy. The continuous increase in the number of functions performed by automatic devices is accompanied by an increase in various types of sensors, converters, calculators, actuators, and other elements of automatic equipment. This, in turn, makes high demands on the parts of aircraft units.

One of the methods of ensuring the compliance of the technical condition of aircraft with the strength requirements, the accepted norms, and rules is the identification of structural and technological defects of the airframe, units, and systems. The solution to the problem of monitoring defects in the structures of

*: Corresponding Author Stakhova Anzhelika, sap@nau.edu.ua

DOI: $10.23890 /$ IJAST.vm02is02.0203 
aircraft and spacecraft is achieved by the results of vibration tests (Berns et al., 2019) (Abdulameer et al., 2015). Such tests are modal control tests of aircraft and dynamic tests, which are an integral part of their ground experimental testing.

When developing new objects of technology, dynamic tests in the process of developing machines, which makes it possible to identify design flaws, and to ensure the safety and quality of its operation, it is necessary to carry out control procedures, diagnostics, and protection, which requires accurate measurements, registration, and analysis of qualitative processes (vibration and shock signals).

Today, it is impossible to name almost a single object of control or production process that would not be affected by vibration, shock, or acoustic loads. The study of oscillatory processes is of great interest for all sectors of the national economy - metallurgy, power engineering, rocket technology, etc. Breakdowns in aviation and space technology are associated with fatigue changes in products under the influence of periodic vibration loads (Berns et al., 2019) (Abdulameer et al., 2015). Therefore, the most common of all types of testing is vibration exposure with a frequency sweep.

Analysis of publications devoted to this topic made it possible to compile a list of defects and establish the causes and consequences of their occurrence. For example, in (Motylev. 2004) (Perera et al., 2009), the appearance of cracks and destruction in a structure is judged by the change in its natural frequencies, forms, and decrements of vibrations. In (Postnov. 2000), to detect damage, it is proposed to use the correction of the inertia and structural stiffness matrices based on changes in the natural frequencies of the system due to the appearance of defects. A method for detecting local damage to composite structures by changing natural frequencies and damping parameters corresponding to various modes of vibration was developed (Perera et al., 2009).

It becomes urgent to solve the problem of further development of methods and creation of devices for recording, analyzing, and controlling dynamic processes to improve the quality of dynamic tests, vibration diagnostics, and vibration protection of machines and equipment at the stage of development and operation. Therefore, in the course of the work, a review and analysis of modern means of measuring vibration and noise according to the vibroacoustic parameters of the working area were carried out. The actual task of design and research of the system of monitoring of vibroacoustic parameters of the working zone in which the influence of vibration and noise parameters is present is set.

\section{Methods}

Today, the number of devices and ancillary equipment measuring vibroacoustic parameters is in the hundreds of types. They differ in accuracy, cost, and availability of different functionality. To ensure the safety of the operation of equipment, it is necessary to carry out periodic diagnostics, which makes it possible to identify incipient malfunctions of machines long before the moment when the failure becomes inevitable, that is, to ensure the operation of equipment according to its technical condition. Due to the huge information capacity of vibroacoustic processes accompanying the operation of machines and the use of computer technology, the requirement to improve the quality and reliability of the operation of machines brings to the fore the methods of vibration diagnostics. Analysis of works in the field of vibration diagnostics (Stakhoca et al., 2021) (Guo et al., 2009) showed that the overwhelming majority of works are devoted to the analysis of the process of generating vibration signals by machine defects, the formation of a reference spectrum and methods for comparing the reference and current spectra. At the same time, in order to improve the quality of vibration diagnostics, especially when diagnosing incipient defects, it is necessary to develop new approaches for developing devices for recording, analyzing, and controlling dynamic processes that improve the quality of dynamic tests, vibration diagnostics, and vibration protection of machines and equipment at the development and operation stage.

When studying vibration parameters, two measurement principles are used - kinematic and dynamic. The kinematic principle is that the coordinates of the points of the object under study are measured relative to the selected fixed coordinate system. The dynamic principle is based on measuring the parameters of the vibration process relative to a stationary frame of reference, in most cases, the centre of gravity of the inertial element of the sensor. In other words, the dynamic principle of vibration measurement is ensured through the use of an inertial mass fixed on an elastic suspension, which, at sufficiently high vibration frequencies, keeps the inertial element practically at rest.

The described measurement principles can be implemented using contact and non-contact vibration measurement methods. When practically any (except induction) vibration sensors are used, the output signals from them do not exceed several tens of millivolts. Therefore, the output of the sensor is connected to the device for amplifying and normalizing the measurement information signal.

\subsection{Sensors for measuring vibration parameters}

At this time, most measurements of vibration processes are carried out using sensors of vibration accelerations 
(accelerometers) (Varanis et al., 2018) (Faisal et al., 2020), and the acceleration is converted into vibration velocity, vibration by electrical methods. This article provides an example of developing a system for measuring vibration parameters using a piezo accelerometer. The device will measure vibration displacement, vibration speed, and vibration acceleration. Another difference is the ability to transmit measurement results over distance. The combination of analog and digital parts allows us to create a device relevant for today, both economically and technically.

The shape and frequency composition of vibration processes is determined by the nature of the excitation forces and the transmission of these excitations to the place where the primary measuring transducer is located. In most cases, the waveform has the form of complex oscillations that contain deterministic and random components of the vibration process. Both the shape and the spectral composition depend on the measured vibration parameter. In practice, displacement, velocity, or acceleration is measured.

Vibration is characterized by the frequency f, i.e., the number of oscillations per second (Hz), amplitude A, i.e., wave offset, or the height of rise from the equilibrium position $(\mathrm{mm})$, speed $\mathrm{V}(\mathrm{m} / \mathrm{s})$ - the speed of the point or system under vibration, and acceleration - acceleration of the point or system under the action of vibration. The absolute values of the parameters that characterize the vibration vary widely, so use the concept of the level of parameters, which is the logarithmic ratio of the value of the parameter to its reference or threshold value.

This device is built using a piezoelectric transducer, which is known to be the most widely used for measuring vibration processes. They surpass all other types of vibration transducers in their technical characteristics because they have advantages: rather high sensitivity, wide frequency, and dynamic ranges of measurements, rather small sizes and weight, high heat resistance, and vibration resistance.

Piezoelectric vibration transducers (VTs) are VTs in which monocrystalline or polycrystalline materials having piezoelectric properties are used as a sensing element (Arnau., 2004) (Ebrahimi.,2011).

Piezoelectric transducers are active transducers that generate a measurement voltage without being energized by an external source. When assessing and predicting the technical state of various objects, piezoelectric accelerometers are used due to their ability to measure vibration in a wide frequency and amplitude range, small overall dimensions and weight, durability in operation, and ease of installation.

The action of piezoelectric VTs is based on the use of a direct piezoelectric effect (Arnau., 2004) (Ebrahimi.,2011), i.e. the properties of some materials to generate a charge under the action of mechanical force applied to them. The principle of construction of piezoelectric VT is shown in Figure 1.

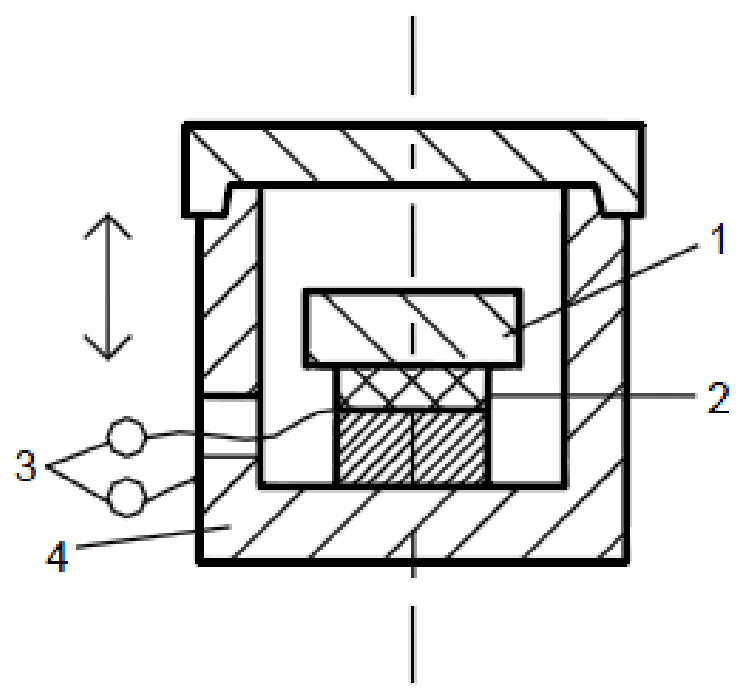

Fig. 1. Scheme of piezoelectric VT

The inertial element 1 is attached to the upper edge of the piezoelectric element 2 , and the lower edge of the piezoelectric element is attached to housing 4 . When installing the transducer on the object under study, the transducer receives the vibration information of the object. Due to the desire of the inertial element to maintain a state of rest, the piezoelectric element is deformed by the influence of the inertial force, where $\mathrm{m}$ is the mass of the inertial element and $a$ is the acceleration of the object.

The deformation of the piezoelectric element and the electric charge that result is proportional to the acceleration. Therefore, these transducers are often called piezo accelerometers.

Poly- and single-crystal piezoelectric substances are used as a piezoelectric element.

The characteristics of the sensing element (SE) depend not only on the value of $\mathrm{m}$ but also on the stiffness $\mathrm{K}$ of the transducer, as well as on the presence of damping (i.e., the resistance to movement along with speed).

The force of elastic $F_{y}$ acting on the inertial mass is known to be equal to

$$
F_{y}=-k x,
$$

where $k$ is the stiffness coefficient, $x$-value mixing.

A characteristic feature of Vibro accelerometers is that they are considered systems with kinematic excitation. In such systems, the measured quantity acts not on the SE but on its base.

Let us introduce two coordinate systems: absolute X, located arbitrarily, and system Z, rigidly connected with the base of the vibration sensor. Then 


$$
\begin{aligned}
& F_{y}=-k(y-k), \\
& F_{d}=-r\left[\frac{d(y-x)}{d t}\right], \\
& F_{i n}=m\left[\frac{d^{2} y}{d t^{2}}\right]
\end{aligned}
$$

where $F_{y}$ is the elastic force, $F_{d}$ is the damping force, $F_{i n}$ is the inertial force, $r$ is the damping coefficient.

According to the d'Alembert principle

$$
F_{i n}=F_{y}+F_{d}
$$

or

$$
m\left[\frac{d^{2} y}{d t^{2}}\right]=-k(y-x)-r\left[\frac{d(y-x)}{d t}\right]
$$

We transform equation (2) to the following form

$$
\begin{aligned}
& \frac{m d^{2} z}{d t^{2}}+\frac{r d z}{d t}+k z=-\frac{m d^{2} x}{d t^{2}}, \\
& z=y-x
\end{aligned}
$$

The quantity $\mathrm{z}$ is the mixing of the seismic mass relative to the sensor body, and $\mathrm{x}$ is the mixing of the sensor body relative to the absolute reference system.

Let vibration mixing $\mathrm{x}$ change according to a harmonic law, then

$$
\begin{aligned}
& \frac{d^{2}}{d t^{2}} \rightarrow-\omega^{2}, \\
& \frac{d}{d t} \rightarrow j \omega,
\end{aligned}
$$

and

$$
-\omega^{2} z(t)+(r / m) j \omega z(t)=\omega^{2} x(t),
$$

where $\mathrm{z}(\mathrm{t})$ and $\mathrm{x}(\mathrm{t})$ are harmonic functions.

If the conditions are satisfied in the last equation $k / m<<\omega^{2} ; r / m<<\omega$, then $z(t) \cong x(t)$. The latter means that the SE operates in the vibrometer mode, i.e., in a steady state, it monitors the amount of vibration mixing. If $r / m>>\omega$ and $r / m>>k / m$, then $(r / m) z(t) \cong-j \omega x(t)$ and the SE also operates in the cycle meter mode, namely, the value $z(t)$ turns out to be proportional to the vibration velocity.

If $k / m>>r / m$ and $k / m>>\omega^{2}$, then this is the operating mode of the vibration accelerometer.

Each of the considered modes can be implemented by choosing the appropriate characteristics of the SE: $\mathrm{m}, \mathrm{k}$, $r$ and the range of operating frequencies. So for a vibrometer, it is important to ensure that the ratios are small $\mathrm{k} / \mathrm{m}$ and $\mathrm{r} / \mathrm{m}$, since it operates in the resonant frequency range. For the velocimeter, it is necessary to use deep damping, and for the accelerometer, it is necessary to use a transducer with great stiffness. In the accelerometer mode, as follows from the above, $r<<k / \omega$ for $\omega<<\omega_{0}, \omega_{0}$ is the natural frequency of the sensor.

\subsection{Accelerometer operation}

Since accelerometers are mainly used in vibrometry, we will analyze their work in more detail. To do this, replace the originals in equation (3) with their images:

$$
\begin{aligned}
& \frac{d}{d t}=p, \\
& \frac{d^{2}}{d t^{2}}=p^{2},
\end{aligned}
$$$$
z(t)=Z(p) ; x(t)=X(p),
$$

where $\mathrm{p}$ is the Laplace operator, and we introduce the following notation, $T=\sqrt{m / k}$ - period of the circular natural frequency of the sensor, $\eta=2 \xi T$ - damping coefficient. Then equation (4) can be represented as follows:

$$
\left(T^{2} p^{2}+2 \xi T p+1\right) Z(p)=-p^{2} X(p) T^{2}
$$

or

$$
W_{1}(p)=Z(p) / p^{2} X(p)=T^{2} /\left(T^{2} p^{2}+2 \xi T p+1\right) .
$$

This expression is the SE transfer function for acceleration, since

$$
a(p)=p^{2} X(p)=\frac{d^{2} x(t)}{d t^{2}} .
$$

The amplitude-frequency $A(\omega)$ and phase-frequency $\psi(\omega)$ characteristics can be obtained by replacing $\mathrm{p}$ with $j \omega$ in the SE transfer function and determining the modulus and argument of the complex frequency spectrum $W(j \omega)$, respectively:

$$
\begin{aligned}
& A(\omega)=W(j \omega), \\
& \psi(\omega)=\operatorname{arctg}[W(j \omega)] .
\end{aligned}
$$

After performing the indicated transformations, we obtain

$$
\begin{aligned}
& A(\omega)=T^{2} / \sqrt{\left(1-T^{2} \omega^{2}\right)^{2}+4 \xi^{2} T^{2} \omega^{2},} \\
& \psi(\omega)=-\operatorname{arctg}\left[2 \xi T \omega /\left(1-T^{2} \omega^{2}\right)\right] .
\end{aligned}
$$

If the admissible value of the dynamic error e is given, defined as 


$$
\delta_{d}=\left[A_{1}\left(\omega_{h}\right)-A_{1}(0)\right] / A_{1}(0),
$$

then the cutoff frequency $\omega_{h}$ of the operating range is found from the condition

$$
1 / \sqrt{\left[\left(1-T^{2} \omega_{h}^{2}\right)+4 \xi^{2} T^{2} \omega_{h}^{2}\right]-1}=\delta_{d} .
$$

The frequency $\omega_{0}$, at which the resonance of the "sensitive element-transducer" system takes place, is called the resonant frequency and is determined by the formula

$$
\omega_{0}=\sqrt{\frac{k}{m}} .
$$

To expand the range of operating frequencies, it is necessary, as follows from (9), to reduce the seismic mass $m$ or to increase the rigidity of its attachment. However, this also decreases the sensitivity of the sensor as a whole since it is proportional to $T^{2}$. Therefore, highfrequency sensors are less sensitive.

An important characteristic of the SE is also its transient impulse response, defined as the inverse Laplace transform of the function $W(p)$ (Li et al., 2016):

$$
K(t)=\frac{1}{2 \pi j} \int_{c-j \omega}^{c+j \omega} W(p) e^{p t} d p
$$

where $K(t)$ is the impulse function, c - abscissa of absolute convergence.

In turn, $W(p)$ can be expressed in terms of the impulse transition function $K(t)$ by applying the direct Laplace transform to the latter:

$$
W(p)=\int_{0}^{n \rightarrow \infty} K(t) e^{-p t} d t
$$

Substituting the function $W(p)$, in formula (10) we obtain

$$
K(t)=-\left[\frac{T}{\sqrt{\left(1-\xi^{2}\right)}}\right] e^{\frac{-\xi t}{T}} \sin \left[\left(\sqrt{\frac{1-\xi^{2}}{T}}\right) t\right] .
$$

It is known that the transient impulse function is the response of the system to the delta action of Dirac (Bahari et al., 2015), i.e., a pulse of infinitely short duration and infinitely large amplitude, the integral parameter of which is equal to one. If a non-periodic signal acts at the input of the system, then in the general case, the response function y $(0$ is determined by the Duhamel integral:

$$
y(t)=\int_{n \rightarrow \infty}^{t} x(\tau) K(t-\tau) d \tau,
$$

where $x(\tau)$ is a function of the input action.

If $x(\tau)$ is a pulse signal of finite duration and amplitude, then at a short duration of this pulse in comparison with the period of the cyclic resonance frequency, the following formula is valid:

$$
\begin{aligned}
& y(t)=S_{0} K(t), \\
& S_{0}=\int_{0}^{t_{n}} x(\tau) d \tau_{i}
\end{aligned}
$$

where $\tau_{i}$ is the pulse duration.

The main advantages of piezoelectric VT are a wide range of operating frequencies, high vibration and shock strength, simplicity of design, low sensitivity to magnetic fields, the ability to create high-temperature transducers, the ability to create transducers with small size and weight.

The main disadvantages of piezoelectric VT are the presence of a large output resistance, dependence of the output signal on the cable length, the inability to measure the constant component of the dynamic process.

\section{Results}

The main goal of the project is to develop a system for monitoring vibroacoustic parameters of the working area.

The main functions of the system are: constant measurement of noise and vibration parameters of the working area, analysis of measured parameters, signalling of exceeding the allowed range of measuring parameters of the working area, display of measured data in decibels.

Based on the review and analysis of technical solutions, the main functions of the system were proposed a block diagram of the system, which is shown in Figure 2.

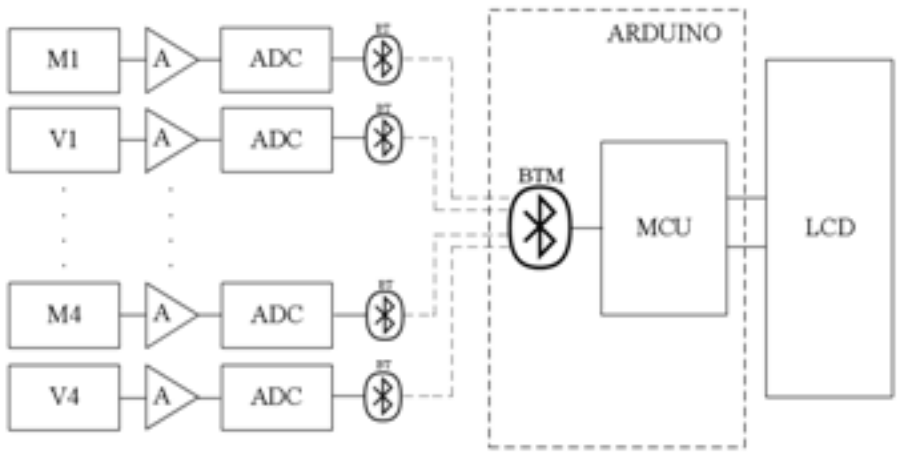

Fig. 2. Block diagram of the MVP system

The diagram shows:

- M1... M4 - microphones (noise sensors); 
- V1... V4 - accelerometers (vibration sensors);

- A - amplifier;

- ADC - analog-digital converter;

- MCU - microcontroller;

- BTM - Bluetooth module for receiving a signal on the mainboard from measuring channels;

- BT - Bluetooth module connected to the sensor to transmit a signal to the mainboard;

- LCD - module for displaying the result.

Figure 1 shows that the system has 8 measuring channels - 4 for vibration measurement and 4 for noise measurement. Each measuring channel consists of the sensor itself (M1-M4 - microphone for noise measurement, V1-V4 - accelerometer for vibration). The data obtained from the sensors are amplified by the operational amplifier A, such amplifiers are 8, for each of the measuring channels. After amplification, the analog signal is converted into discrete code using a singlechannel ADC. The digital signal after the ADC is transmitted to the mainboard with a microcontroller. The board receives data from all eight measuring channels. The results of collecting the values obtained from the sensors, the mainboard uses software processing to analyze the data and transmits them to the LCD display, which in turn displays them.

In the system, we propose to use the board "Arduino UNO" (Fig. 3). The main characteristics are shown in table 1.

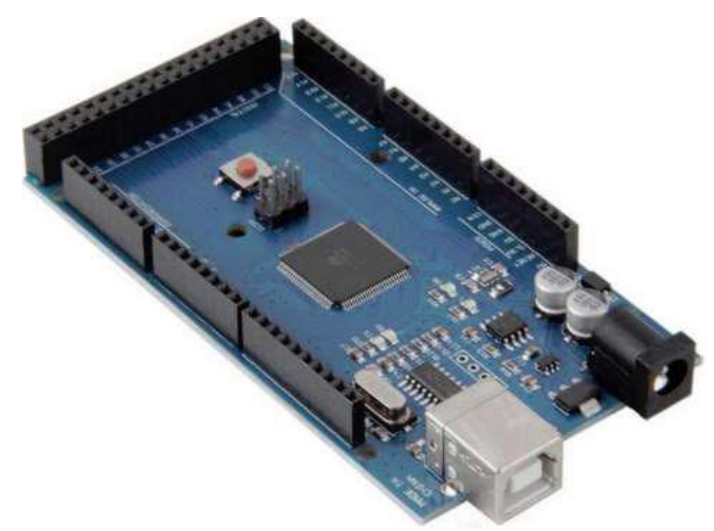

Fig. 3. General view of the ARDUINO UNO board

Table 1. Characteristics of the ARDUINO mainboard (Components101, 2021)

\begin{tabular}{ll}
\hline Microcontroller & ATmega 328 \\
Supply voltage & $7-12 \mathrm{~V}$ \\
Number of channels & 8 \\
Clock frequency & $16 \mathrm{MHz}$ \\
Operating voltage & $5 \mathrm{~V}$ \\
Flash memory & $32 \mathrm{~KB}$ \\
Digital inputs / outputs & 14 \\
\hline
\end{tabular}

As can be seen from Table 1, the ARDUINO board has eight input channels, which will allow us to process, analyze, and transmit data, and we, in turn, will use four channels for noise measurement and four channels for vibration. The use of multichannel during measurement will increase the accuracy of the obtained data. Since the board has a "Flash-memory," we will use it as a data warehouse for a given period, which will allow us to analyze the measured data for a given period of time.

A microphone is used as a sensor for each noise measurement channel. The analog signal from the microphone is amplified by an amplifier and converted into digital code by an ADC. Then the digital signal is transmitted to the mainboard with a microcontroller.

Noise parameters are measured by a sensor (microphone), and then the analog signal is fed to the amplifier. These two elements, the sensor and the amplifier, are located on the SFM board. Then the amplified analog signal is fed to a single-channel ADC (MCP3421), where it is converted into discrete code and can be amplified as needed 2, 4, or 8 times. The sampled signal is then sent to the Bluetooth module, then transmitted to the same Bluetooth module, but all connected to the ARDUINO mainboard.

Similarly, an accelerometer is used for each parameter measurement channel. On each measuring channel, both for vibration parameters and for noise parameters, the analog signal received from the sensor is amplified, sampled, and transmitted to the microcontroller.

Vibration parameters are measured by a three-axis accelerometer (sensor), and then the measured analog signal is amplified. As well as for measuring noise parameters, the sensor and amplifier are on the same board - SW420. The signal is then converted to digital code using the same ADC (MCP3421) and transmitted to the ARDUINO mainboard using the same Bluetooth module as for the noise settings.

There are eight measuring channels of vibroacoustic parameters, 4 of them for noise parameters and 4 for vibration parameters.

Digital signals from measuring channels by means of the Bluetooth module on each of the channels are transferred to payment through the same Bluetooth module.

On the board itself, the microcontroller contains a calibration characteristic used to analyze the received measurement signals, and then the processed data is displayed on the LCD display. Depending on whether the obtained values exceed the normal ranges of human work in such an environment, there may be an alarm about the excess.

In the system of monitoring of vibroacoustic parameters, data transfer from sensors to a processing board will be 
carried out by means of the Bluetooth module. A Bluetooth module will be connected to each measuring channel to transmit amplified and sampled data to the board. Such a module will also be connected to the board to receive a signal from the measuring channels.

The measuring channels of the designed system transmit the measured data via a wireless connection, which simplifies their use and placement in any place in the work area and allows to cover a larger area of the measured area. Analogs are portable devices that measure certain small planes of the working area.

As for the principles of operation, despite a large number of devices, the principle of their operation remains unchanged. The principle of measuring noise parameters is based on the received acoustic waves with the subsequent conversion of their energy by means of the most various technologies in electric potential, which is directly proportional to the size of a signal. As for the principle of measuring vibration parameters, vibration sensors are used, which perceive mechanical vibrations, which are also converted into the corresponding electric potential by means of various technologies.

The system of monitoring of vibroacoustic parameters performs the following functions:

- measurement of noise and vibration parameters;

- analysis of measured data;

- check for exceeding the allowed range of work;

- indication of measured data;

- alarm when exceeding the permitted operating range.

Since the measurement data coming from the sensors are not discretized, our system provides for the sampling of this data using an ADC. Calibration is performed for the normal display of the measured signal on the LCD display, so the previously found calibration characteristic will be entered on the ARDUINO mainboard. Also, as a system setting, it is possible to set the time of the sensor interrogation, i.e., with what interval the measured data from the measuring channels will be received on the mainboard.

As well as calibration characteristics, in the mainboard, namely in the microcontroller, the maximum values of ranges for parameters of noise and vibration for the analysis of the received measuring data will be entered.

After the analysis, depending on the result, an alarm will be performed to exceed the range, if any. The data will then be stored in the flash memory of the microcontroller. The last step is to display the measured data on the LCD display.

Therefore, taking into account all the functions of the system, the developed algorithm of the vibroacoustic parameters monitoring system is presented in Figure 4

Consider the developed algorithm of the system. As can be seen from Figure 4, after the initialization of the systems, calibration characteristics are entered to display the obtained sampled signal from the measurement channels in decibels.

Then, all measuring channels are connected to the mainboard and enter the survey frequency of these channels, namely the frequency of data acquisition.

After that, the connection of the measuring channels with the ARDUINO board is checked, if the connection is not stable, we return to the connection setting, but if it is stable, we start measuring the data.

The first stage of measurement is to check the set input frequency of interrogation of measuring channels. At the first initialization of the system, this stage is skipped because the mainboard has not yet received the measured data, so we immediately proceed to the survey of measurement channels. If the data during the survey of measuring channels is not received, then we return to the check of the set frequency. Upon successful survey of measuring channels and obtaining data, the analysis of these data is performed. The analysis is followed by a comparison with the maximum allowable values for workplace work for noise and vibration parameters. If the data exceeds, then the signal is exceeded, and after entering the received data in the flash memory of the microcontroller, if the data does not exceed the maximum allowable values, the data is saved.

\section{Conclusions}

Solving the problem of identifying aircraft defects using the changes in the dynamic characteristics of the controlled objects caused by them, the article proposed a system for monitoring the vibroacoustic parameters of the working area, which performs the following functions: measurement of vibroacoustic parameters (vibration and noise parameters) of the working zone; analysis of measured values of vibroacoustic parameters; alarm of the user about exceeding of the allowed range of parameters of vibration and noise of a working zone; saving the measured data for a specified period of time; display of measured data.

The use of the developed system for monitoring vibroacoustic parameters will allow detecting such types of defects as a violation of the integrity of structures, loosening of fasteners and the appearance of gaps in the joints of units, backlash in mechanical systems for transferring forces or displacements, increased dry friction in the supports of deflected surfaces, resonant modes of vibration of elements and systems airframe, insufficient efficiency of hydraulic dampers in the elastic 
airframe.

The developed system has a number of advantages due to the selected technical solutions, namely a sensor for measuring vibroacoustic parameters, which meets the needs of the task and has the following advantages: large measurement range, low noise, signal frequency range, signal-noise ratio. The use of ADC in the system allows the selection of the sampling frequency of the signal programmatically. Also, the advantage of the system is the use of a Bluetooth module, which has a long-range of signal reception, which will allow us to place measuring channels in any desired place in the work area, and the board has a high level of data protection. The measuring channels of the developed system transmit the measured data by means of a wireless connection, which simplifies their use and placement in any place of the object of control and allows covering a larger area of the measured area.

\section{Abbreviations}

\begin{tabular}{ll}
\hline VTs & vibration transducers \\
MCU & microcontroller \\
A & amplifier \\
M & microphones \\
V & accelerometers \\
LCD & module for displaying the result \\
ADC & analog-to-digital converter \\
SFM & SparkFunMicrophone \\
BTM & Bluetooth module for receiving a signal on the \\
& mainboard from measuring channels \\
BT & Bluetooth module connected to the sensor to \\
& transmit a signal to the mainboard \\
\hline
\end{tabular}

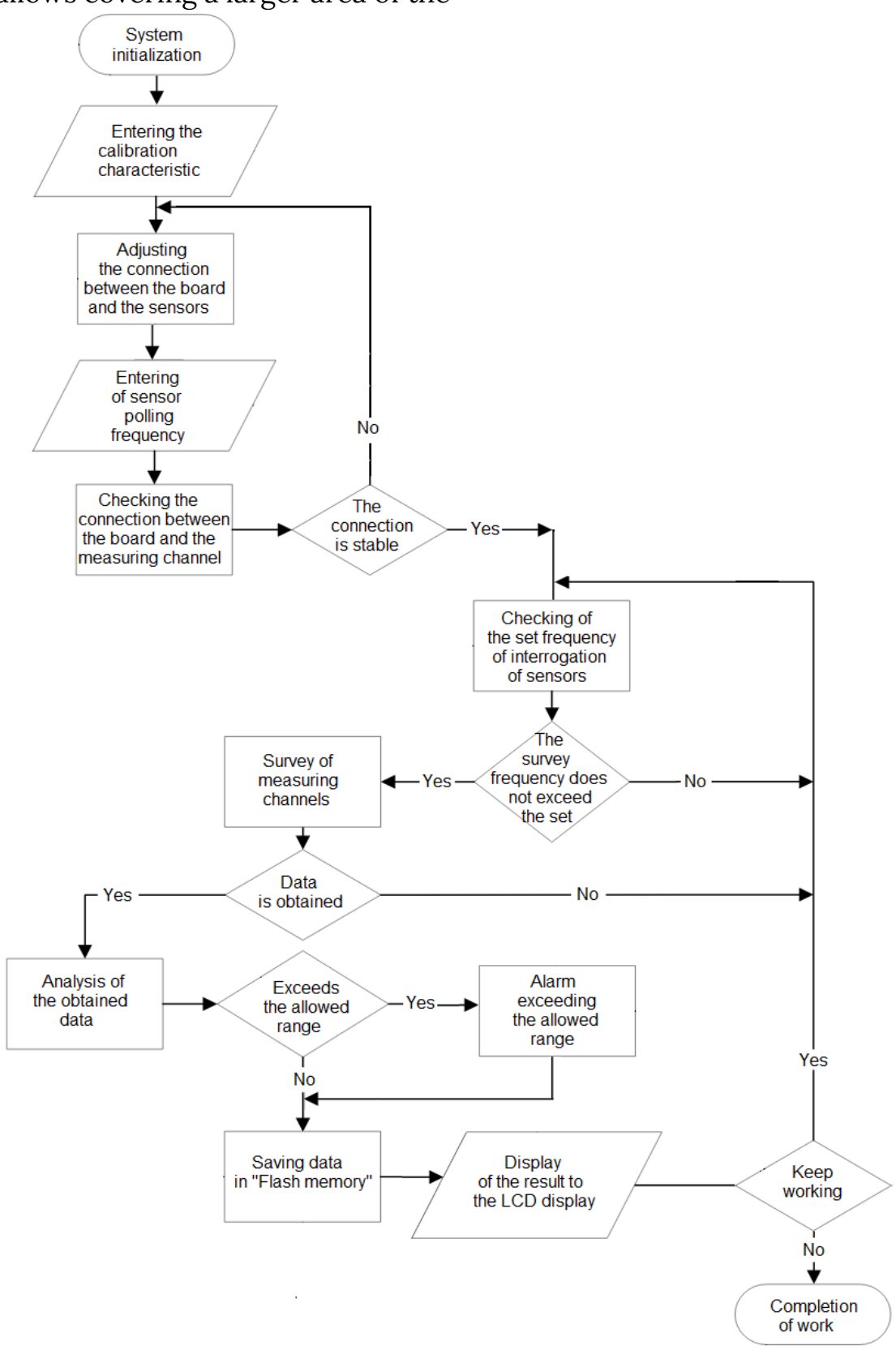

Fig. 4. Algorithm of measurement system operation 


\section{References}

Abdulameer, H.A. and Wasmi, H.R., 2015, Vibration control analysis of aircraft wing by using smart material, Innovative Systems Design and Engineering, 6(8), pp.7-42.

Advanced Avionics Handbook. - U.S. Department of Transportation, FAA, 2009, - 112 p.

Arnau, A. Ed., 2004, Piezoelectric transducers and applications, Berlin: Springer.

Bahari, A. and Popplewell, N., 2015, Comment on "Transient response of an acoustic medium by an excited submerged spherical shell" [J. Acoust. Soc. Am. 109 (6), 2789-2796 (2001)], The Journal of the Acoustical Society of America, 137(5), pp.2966-2969.

Berns, V. and Zhukov, E., 2019, Diagnostics of airframes defects by vibration tests results, In AIP Conference Proceedings (Vol. 2125, No. 1, p. 030089), AIP Publishing LLC.

Components101, 2021, Arduino Uno, Available at: https://components101.com/microcontrollers/ar duino-uno, (Accessed 3 August 2021).

Da Silva, R.R., Costa, E.D.S., De Oliveira, R.C. and Mesquita, A.L., 2017, Fault diagnosis in rotating machine using full spectrum of vibration and fuzzy logic, Journal of Engineering Science and Technology, 12(11), pp.2952-2964.

Ebrahimi, F. ed., 2011, Advances in piezoelectric transducers, BoD-Books on Demand.

Faisal, I.A., Purboyo, T.W. and Ansori, A.S.R., 2020, A review of accelerometer sensor and gyroscope sensor in IMU sensors on motion capture. Journal of Engineering and Applied Sciences, 15(3), pp.826829.

Guo, L., Chen, J. and Li, X., 2009, Rolling bearing fault classification based on envelope spectrum and support vector machine, Journal of Vibration and Control, 15(9), pp.1349-1363.

Iwaniec, M., Holovatyy, A., Teslyuk, V., Lobur, M., Kolesnyk, K. and Mashevska, M., 2017, Development of vibration spectrum analyzer using the Raspberry Pi microcomputer and 3-axis digital MEMS accelerometer ADXL345, In 2017 XIIIth International Conference on Perspective Technologies and Methods in MEMS Design (MEMSTECH) (pp. 25-29). IEEE.

Jarmołowicz, M. and Kornatowski, E., 2017, May. Method of vibroacoustic signal spectrum optimization in diagnostics of devices. In 2017 International Conference on Systems, Signals and Image Processing (IWSSIP) (pp. 1-5). IEEE.
Li, J., Farquharson, C.G. and Hu, X., 2016, Three effective inverse Laplace transform algorithms for computing time-domain electromagnetic responses. Geophysics, 81(2), pp.E113-E128.

Motylev, NI, 2004, Identification of mechanical defects in the elements of reactors and structures of nuclear power plants under test vibration impact, Technologies and systems for ensuring the life cycle of nuclear power plants, SPb .: Mendeleev, (2), pp. 126-131.

Perera, R., Fang, S.E. and Huerta, C., 2009, Structural crack detection without updated baseline model by single and multiobjective optimization, Mechanical Systems and Signal Processing, 23(3), pp.752-768.

Postnov, VA, 2000, Determination of damages of elastic systems by mathematical processing of frequency spectra obtained from the experiment, Izv. RAS. Rigid Body Mechanics, (6), pp. 155-160.

Rupitsch, S.J., 2018, Piezoelectric sensors and actuators: Fundamentals and applications, Springer.

Stakhova, A. and Kvasnikov, V., 2021, Development of a device for measuring and analyzing vibrations. Informatyka, Automatyka, Pomiary w Gospodarce i Ochronie Środowiska, 11(2), pp.48-51.

TOMASZEK, H., STĘPIEŃ, S. and WAŻNY, M., 2010, Aircraft flight safety with the risk of failure during performance of an aviation task, TRIBOLOGY• RELIABILITY• TEROTECHNOLOGY DIAGNOSTICS• SAFETY• ECO-ENGINEERING TRIBOLOGIA• NIEZAWODNOŚĆ• EKSPLOATYKA DIAGNOSTYKA• BEZPIECZEŃSTWO• EKOINŻYNIERIA, p.75.

Varanis, M., Silva, A., Mereles, A. and Pederiva, R., 2018, MEMS accelerometers for mechanical vibrations analysis: A comprehensive review with applications, Journal of the Brazilian Society of Mechanical Sciences and Engineering, 40(11), pp.1-18. 